\title{
Strates
}

STRATES Matériaux pour la recherche en sciences sociales

5 | 1990

Conjuguer stratégies et territoires?

\section{Systèmes de pouvoir et gestion du territoire dans le sud du Parc national des Cévennes}

\section{Marie-Claude Guerrini}

\section{(2) OpenEdition}

1 Journals

Édition électronique

URL : http://journals.openedition.org/strates/1440

DOI : $10.4000 /$ strates. 1440

ISSN : $1777-5442$

Éditeur

Laboratoire Ladyss

Édition imprimée

Date de publication : 31 décembre 1990

ISSN : 0768-8067

Référence électronique

Marie-Claude Guerrini, « Systèmes de pouvoir et gestion du territoire dans le sud du Parc national des Cévennes », Strates [En ligne], 5 | 1990, mis en ligne le 16 mars 2007, consulté le 08 septembre 2020. URL : http://journals.openedition.org/strates/1440; DOI : https://doi.org/10.4000/strates.1440

Ce document a été généré automatiquement le 8 septembre 2020.

Tous droits réservés 


\title{
Systèmes de pouvoir et gestion du territoire dans le sud du Parc national des Cévennes
}

\author{
Marie-Claude Guerrini
}

1 La recherche s'attache à l'étude des systèmes de pouvoirs, c'est-à-dire les interrelations et interactions entre les composants d'un système pour la gestion d'espaces définis, délimités et désignés dans le cadre d'une politique de l'État, celle de l'environnement.

2 Les Parcs nationaux sont en effet une des expressions de cette politique de protection de la nature définie à partir de 1960. Par la promulgation et l'application de textes de lois, l'État peut mettre sous tutelle un «milieu naturel [qui] présentée un intérêt spécial et qu'il importe de préserver contre tout effet de dégradation naturelle et de [le] soustraire à toute intervention artificielle susceptible d'en altérer l'aspect, la composition et l'évolution ». Le texte met tout d'abord l'accent sur l'aspect protection et conservation: ceci pourrait conduire à une politique de type muséographique consistant à isoler des enjeux sociaux un milieu «naturel » dans lequel l'écosystème évoluerait à l'abri de toute intervention humaine. Mais le territoire désigné pour devenir un Parc est anthropisé, utilisé et approprié par d'autres acteurs privés, collectifs, voire par l'État lui-même.

3 Le Parc national des Cévennes (P.N.C.), créé en septembre 1970, couvre un territoire de 91000 hectares, comprenant plus de $63 \%$ de terrains privés. Un Établissement public à caractère administratif assure l'aménagement, la gestion et la réglementation du Parc dont les principes sont définis par le Conseil d'administration qui délibère sur un Programme d'aménagement sur cinq ans et vote le budget.

4 La démarche consiste en une identification des acteurs qui forment le système en regard des pratiques, au nombre de trois, que l'on trouve dans le P.N.C. :

5 - pratiques à des fins de production, représentées ici essentiellement par l'activité pastorale sur les Hautes Terres et des systèmes de production plus diversifiés dans le Viganais, et par l'exploitation forestière ; 
les conséquences territoriales de leurs actions. Il s'agit en effet non seulement de les contradictions, voire les risques de conflits, nés de leurs interrelations, à l'intérieur du système qu'ils forment. Il faut cependant souligner que les systèmes formés seront différents, selon que l'on examine le rôle et les pratiques des divers intervenants dans la zone centrale du Parc (où le Parc représente la seule et unique autorité en matière d'aménagement), ou dans la zone périphérique (où tous les intervenants sont à même de mettre en œuvre des stratégies, même si leur poids et leur capacité d'intervention sont inégaux). C'est en fonction des pratiques que les différents acteurs vont élaborer ou non des stratégies, afin de parvenir à leur but. C'est en analysant les comportements que l'on peut poser la question de savoir s'ils relèvent et révèlent des stratégies : s'il est parfois possible de détecter les stratégies qui sous-tendent les actions, il est souvent difficile de les analyser, soit parce qu'elles ne sont pas clairement affichées, soit parce que les groupes sociaux, a fortiori les individus, n'en ont pas, soit enfin parce que, plutôt que de stratégies, on doit parler de tactiques au coup par coup, de réactions, d'opposition à des comportements ou à des décisions que le groupe juge hostiles à son égard.

9 On peut tenter une première analyse des comportements du système en insistant sur ce qui représente à nos yeux une stratégie pour la gestion du territoire.

Les gestionnaires institutionnels

10 * Le Parc national des Cévennes a tenté d'allier la défense écologique et la rénovation rurale, de mener une politique de conservation et de développement. Ses interventions sont importantes et il joue un rôle moteur dans le maintien et le développement du système agro-pastoral. La région cévenole est très dévitalisée, la population souvent vieillie, et il est important de maintenir une population agricole qui diminue sans cesse. Un des buts du Parc est de soutenir ces activités agropastorales qui permettent l'entretien du milieu et qui, bien conduites, n'entraînent pas de dégradations. En zone centrale, il a pratiqué dans le secteur Aigoual-Lingas une politique foncière par l'intermédiaire de la Société d'aménagement foncier et d'établissement rural (SAFER), rachetant deux propriétés mises en fermage et des terrains de parcours loués pour la transhumance à la Coopérative d'Estive de brebis raïole. Il intervient aussi en attribuant des aides aux exploitants agricoles par les plans d'environnement, et en participant à la protection du patrimoine génétique d'espèces animales ou de variétés végétales locales (brebis raïole, cheval meyrens, mûriers, châtaigniers...).

Dans la zone périphérique, le Parc n'intervient plus en tant que gestionnaire direct, en particulier par acquisition de biens fonciers, mais soutient au même titre que d'autres administrations des actions de développement rural et d'aide au maintien des exploitations agricoles.

12 L'une des premières missions du Parc est d'assurer la protection de la nature et de travailler à la remontée biologique de la faune et de la flore : il a ainsi procédé à la 
réintroduction d'espèces (cerfs, chevreuils, tétras-lyres, vautours, par exemple), et créé des réserves intégrales, sous-trayant ainsi des territoires boisés, de superficie limitée, à toute intervention humaine (Forêt du Marquairès, Vallée de la Brèze).

13 Cette mission de protection conduit le Parc à exercer un pouvoir "culturel ", en organisant l'éducation du public. En effet, s'il admet la fréquentation touristique de son territoire, il voudrait cependant un touriste «intelligent » et surtout "éduqué ». Il s'efforce de faire passer une «certaine vision » de la nature, que l'on doit connaître pour la respecter et éviter toute dégradation. Des centres d'information, des séances d'animation et des promenades guidées par les gardes-moniteurs attestent de ce souci pédagogique.

Dans la mesure où les prérogatives du Parc en matière de réglementation sont quasi exclusives, au moins en zone centrale, on peut se demander quelles forces peuvent avoir d'autres pouvoirs et par quoi ils sont représentés. Certains exercent un pouvoir direct de gestion de l'espace, d'autres se présentent comme des groupes de pression ; ils forment avec le Parc un système de relations et d'interactions influençant la dynamique de ces espaces. Il faut en effet souligner que la majeure partie du foncier appartient soit à des propriétaires privés, soit à l'État, géré par un autre Établissement public, l'office national des forêts.

15 * L'Office national des forêts est le deuxième "pouvoir » étatique dans la zone : la forêt de l'Aigoual, œuvre des forestiers depuis la fin du XIX siècle, couvre 15555 hectares et se trouve incluse dans sa quasi totalité dans le Parc. L'État, qui est propriétaire des terrains des forêts domaniales administrées par l'O.N.F., n'a pas acquis l'ensemble du territoire géré par le Parc. Se présentant eux-mêmes comme les premiers protecteurs de l'environnement, les forestiers gèrent cependant l'écosystème forestier à des fins de production, l'office étant un Établissement public industriel et commercial qui tire une partie de ses ressources des ventes de bois. Les plans d'aménagement répondent à cette logique d'une politique de production et ne sont pas toujours en conformité exacte avec les souhaits du Parc qui, en particulier, serait pour le maintien ou la création d'espaces ouverts dans le massif forestier, nécessaires à la faune sauvage. Des risques de conflits concernent l'ouverture et le libre accès des routes forestières fréquentées par les automobilistes de passage de façon très ponctuelle.

Dans le cas de ces deux intervenants institutionnels qui agissent en fonction d'une politique clairement définie et affichée, l'une de protection de la nature, l'autre de production de biens marchands, on peut dire qu'ils définissent des stratégies qui leur permettent d'atteindre le but fixé. Si la stratégie foncière, donc territoriale, est évidente dans le cas de l'O.N.F. qui doit se constituer un territoire de production, elle est moins nette pour le Parc qui n'a acquis un patrimoine foncier que pour tenter de perpétuer des systèmes d'exploitations en voie de disparition. La politique qui consiste à aider les exploitants pour les maintenir sur place demande pour le Parc la définition d'une stratégie financière adaptée, soit sans cesse réajustée en fonction des lois du marché qui obligent les exploitants à adapter leurs productions, soit camouflant une subvention à des « jardiniers de la nature ».

$17{ }^{*}$ Les collectivités locales et les administrations: les limites du Parc recoupent des unités administratives, les communes. Sa création a suscité de vives oppositions. Les élus locaux saisissaient mal les buts recherchés, estimant qu'il s'agissait d'une atteinte à leur pouvoir sur le territoire communal et pensant que les populations locales avaient toujours su "protéger la nature », sans qu'une autorité supérieure ne soit chargée de 
réglementer ce qui pouvait être du ressort des compétences des élus locaux. Les Conseils municipaux des communes incluses dans la zone centrale ont voté en majorité contre l'installation du Parc (13 communes sur 15 ont voté non dans la zone AigoualLingas). Ils souhaitaient conserver une totale liberté pour mener la politique d'aménagement conduisant à un développement local, même s'il s'agissait le plus souvent de s'appuyer sur une monoactivité qui ne prenne qu'indirectement en compte la gestion de la nature elle-même.

Certains préfèrent mettre l'accent sur le maintien et le développement d'activités (artisanat et industrie) qui n'ont pas de liens directs avec le milieu naturel. Même si beaucoup d'élus locaux déplorent «l'abandon» du milieu et son non-entretien, ils comptent bien asseoir le développement de leur commune sur la création d'activités liées à la fréquentation de la nature (le tourisme), sans pour autant poser la question de la « qualité » de la nature offerte aux touristes. Cette stratégie de développement local basée sur le tourisme risque de provoquer des conflits avec le Parc qui n'admet, en principe, aucune installation autre que celle des exploitants agricoles. Le pouvoir des élus, pour les parties de leur commune situées en zone centrale, est très faible; certains ont cependant réussi à peser suffisamment pour obtenir l'extension de pistes de ski aménagées dans le Parc.

19 Dans la zone périphérique, où les rapports de force sont assez différents et se situent au niveau normal de tout aménagement, diverses volontés politiques locales se sont exprimées et traduites par des actions visant à mieux gérer les territoires communaux dans le but d'un développement local.

20 En 1975, un Plan d'aménagement rural (PAR) comprenant les cantons du Vigan, de Valleraugue, de Trèves, de Sumène et d'Alzon, reçoit l'agrément du ministère de l'Agriculture. À la suite du Schéma directeur de la zone périphérique du Parc des Cévennes, le Plan est basé sur le maintien d'une population active permanente fondée sur le développement de l'agriculture. Si la part des investissements agricoles a été faible, les exploitants ont cependant bénéficié de soutiens pour certains aménagements ou réfections de systèmes d'irrigation, ou pour entreprendre des améliorations pastorales (remise en culture herbagère, pose de clôtures, complétées par des aménagements fonciers). Dans le PAR, les conflits pour l'utilisation de l'espace ont été abordés par divers documents d'aménagement : Zone à environnement protégé (ZEP) à Mandagout et Arphy, Plan d'occupation des sols (POS) au Vigan et pour l'Espérou, qui relève des communes de Dourbies et de Valleraugue, et Plans paysagers pour lesquels sont concernés la Direction départementale de l'Agriculture, la Direction départementale de l'Équipement et l'Atelier d'aide architecturale, ont pour but de sensibiliser les élus locaux et les habitants permanents à la préservation des espaces naturels et à la mise en valeur, ainsi qu'à la qualité des espaces constructibles et des constructions ; on approche ainsi d'un zonage indicatif des sols au niveau agro-sylvopastoral, et de la détermination de zones constructibles dans des sites choisis. Une dizaine de communes possèdent actuellement leur Plan paysager.

21 En 1984 est signée une convention pour la mise en place d'une Charte intercommunale du canton du Vigan. Dans les projets, deux axes concernant le milieu et sa gestion ressortent nettement :

22 - il est prévu de réaliser une carte foncière présentant des données pour caractériser l'état du parcellaire et les besoins des exploitants agricoles: modes de faire-valoir, besoins d'extension, sous-utilisation, potentialités de terres agricoles. Cette carte doit 
permettre un dialogue entre les agriculteurs et les propriétaires qui n'utilisent plus eux-mêmes le milieu.

- le secteur forestier (forêt privée) doit être pris en compte pour aboutir à une revalorisation du capital forestier. Dans le canton du Vigan, la forêt privée est très morcelée : depuis vingt-cinq ans, 103 personnes physiques ou morales ont bénéficié d'aides pour aménager au total 807 hectares. Elle n'a jamais fait l'objet d'une politique de massif comme la forêt domaniale de l'Aigoual, aujourd'hui réellement productive ; elle nécessite cependant la définition d'une politique forestière et le développement d'actions mobilisant les propriétaires forestiers et visant la revalorisation de la forêt et son exploitation. Il faudra recourir à un zonage afin de préciser les différentes vocations des terrains concernés : sylviculture, agriculture, pastoralisme, tourisme.

24 À un niveau plus large, concernant la Charte intercommunale du Vigan, celle de Meyruels- Sainte Enimie et les cantons de Trèves et Alzon, est mis en place un Pays d'accueil pour le développement touristique de la région Causses-Aigoual-Cévennes. Ce développement sera fondé sur le renforcement du "tourisme vert » reposant sur les ruraux et principalement les agriculteurs (accueil à la ferme, gites ruraux) et sur la promotion d'animations liées à l'initiation à l'environnement et à la découverte du milieu.

Les pouvoirs représentés par les gestionnaires institutionnels - État et Collectivités locales - tendent à concevoir des projets de développement s'appuyant de façon plus ou moins évidente sur les forces locales, certains intégrant avant tout les contraintes liées à la protection de la nature et le maintien des équilibres écologiques, favorisant certaines pratiques du milieu. Ils doivent cependant compter avec d'autres composantes du système qui possèdent et exercent, ou veulent exercer, un pouvoir dans l'aménagement ou l'usage du milieu, possible source de conflits.

Les propriétaires et les usagers

C'est essentiellement dans la zone centrale du Parc que les risques de conflits sont les plus importants, en particulier avec l'Établissement public qui la maintient sous contrôle.

Les propriétaires

Ils sont un élément important du système car ils possèdent plus de $60 \%$ des terres situées dans la zone centrale du Parc et peuvent élaborer des stratégies contraires à la politique mise en place par l'État et visant à contrôler, voire interdire, toute activité qui n'a pas pour but d'utiliser l'espace à des fins de production agro-pastorale, ou éventuellement, avec son accord, sylvicole. Cependant, les représentations de l'espace et les usages qui en sont faits sont très différents suivant les propriétaires : ceux-ci représentent en effet à la fois le niveau interne et englobant du système, selon qu'ils sont résidants ou absentéistes.

Parmi les propriétaires résidants, les exploitants agricoles utilisent l'espace à des fins de production selon des modes d'exploitation de type extensif, surtout dans les zones où l'élevage est le principal système de production. Leur nombre a diminué de façon importante : - $58 \%$ entre 1970 et 1980, avec une diminution moins accentuée dans les communes lozériennes (- $37 \%$ ). Les Surfaces agricoles utilisées (SAU) augmentent dans certaines communes, signe de la reprise d'une fraction des terres par les exploitants restants, mais les agriculteurs ont beaucoup de mal à mettre en œuvre leur stratégie foncière, se heurtant aux autres acteurs fonciers : l'usage qu'ils veulent faire de la terre laisse de moins en moins de place à l'agriculture. Les agriculteurs vont se heurter aux 
propriétaires dont l'attachement à la terre est renforcé par les espoirs de spéculations sur les terrains à bâtir, le développement des résidences secondaires permettant une plus-value sur des terres souvent bien situées, par exemple en fond de vallée et occupées le plus souvent par des prés nécessaires aux éleveurs. Lorsqu'il est possible aux exploitants d'obtenir des terres en location, encore hésitent-ils à entreprendre des travaux d'aménagement, ne sachant pas la durée exacte de la jouissance dont ils disposent. Mais cette situation n'est envisageable que hors des limites de la zone centrale du Parc, essentiellement dans le Viganais. Sur les terres incluses dans celles-ci, c'est-à-dire surtout sur les Hautes Terres où la spéculation est théoriquement impossible, la réglementation interdisant tout usage autre qu'agricole, le marché foncier n'est pas beaucoup plus libre. On peut alors poser la question du devenir des terres qui seront laissées pour compte lorsque les exploitations agricoles auront atteint les limites de leurs possibilités d'extension. Châtaigneraies abandonnées, faute de main-d'œuvre pour les entretenir, friches envahies par les genêts et les broussailles, évoluant vers les boisements spontanés, marquent actuellement fortement le paysage. La question de « l'entretien de la nature » est à l'ordre du jour dans cette zone : elle a été posée par le Parc qui a tenté d'y répondre en proposant aux exploitants des contrats (contrats Mazenot) pour qu'ils assument cette tâche.

Si les agriculteurs possèdent un réel pouvoir dans le processus évolutif des paysages et du milieu naturel, ils ont de plus en plus de mal à l'exercer sans soutier et reconnaissance de leurs productions et, à ce propos, on doit aussi s'interroger sur l'efficacité de la Loi Montagne applicable à cette zone en sus des aides et subventions du Parc. Il faut alors s'interroger sur la stratégie que les exploitants seraient capables de mettre en œuvre pour acquérir des terres qui leur permettraient d'obtenir un rendement maximum, sans dépasser les capacités d'autorégulation de leur entreprise.

Les propriétaires non-exploitants, résidants ou non - en particulier sur les Hautes Terres - ont des attitudes différentes. La majorité d'entre eux ont une stratégie de résistance et n'aliènent que très peu le patrimoine familial qui sera divisé en autant d'ayants droit lors des successions. Certains cependant louent, le plus souvent par accord verbal, leurs terres pour une utilisation agropastorale. D'autres tentent de donner une plus-value à leurs propriétés en les reboisant, ce qui transforme radicalement l'écosystème, ou les vendent comme terrains à bâtir pour des résidences principales ou secondaires. Dans ce dernier cas, le problème est aigu lorsque ces terrains se trouvent en zone Parc. Les propriétaires se heurtent directement aux contraintes paysagères et architecturales imposées par la réglementation du Parc et en particulier à l'interdiction de construire d'autres bâtiments que ceux destinés à l'exploitation agricole.

31 Pour lutter contre ces contraintes et ces interdictions, une Association de défense des propriétaires du Parc s'est créée: "Solidarité caussenarde et cévenole ». Groupe de pression important, surtout implanté dans la zone d'Aigoual, cette association rassemble des propriétaires de la zone centrale; elle demande la suppression, ou tout au moins la transformation, du Parc national, veut que l'on reconnaisse aux propriétaires le droit de gérer leurs terres comme ils l'entendent et s'élève contre la réglementation imposée par le Parc et les dégâts causés par la faune sauvage sur les terres des propriétaires; elle développe une stratégie visant à faire annuler les décrets consécutifs. 
32 En dehors de la zone Parc, en l'absence de Plan d'occupation des sols (POS), les propriétaires ont souvent une attitude qui renforce la concurrence entre les terres agricoles et les terrains à bâtir, pouvant entraîner des conflits entre propriétaires et exploitants cherchant à s'agrandir.

Les usages non-propriétaires

33 1. Les transhumants sur les Hautes Terres, par leur pratique et leur gestion du milieu, se rapprochent des exploitants, sans en avoir la capacité foncière, se heurtent comme eux à l'immobilisme en matière de marché foncier, et subissent le contrecoup des espoirs de spéculation sur les terres agricoles qui ne sont pas pour l'instant des terres constructibles. Le changement d'orientation de la transhumance (diminution de la transhumance ovine et augmentation de la transhumance bovine) a entraîné de profonds changements dans l'utilisation de l'espace. Les clôtures, nécessaires aux parcs à bovins, fragmentent l'espace, bloquant parfois à des troupeaux ovins l'accès à des parcours qui ainsi ne sont plus utilisés dans leur totalité ; il se produit une accélération de l'embroussaillement par suite de la non ou sous-utilisation de certaines parcelles. Les troupeliers ont perdu une grande partie de leur rôle social et la transhumance ovine dépend maintenant fortement du P.N.C. qui a racheté des terrains pour maintenir les parcours.

34 2. Pour d'autres usagers qui participent de façon indirecte à la gestion de l'espace, la nature offre la possibilité d'un prélèvement de produits : la cueillette et la chasse sont deux activités qui ont une grande importance. Dans les limites du Parc, les droits d'usage sont en principe réservés aux propriétaires et aux ayant droit. La cueillette est peut-être pratiquée à moins grande échelle que dans d'autres zones du Massif central ; elle représente cependant un prélèvement assez considérable, et par là même un revenu non négligeable. En automne, les forêts domaniales sont envahies de cueilleurs ; la plupart d'entre eux sont des promeneurs, amateurs, et vont un peu au hasard des promenades; Certains cependant sont de véritables professionnels et, sans gérer la forêt, en font un territoire qu'ils contrôlent de façon indirecte, en ne prélevant qu'en certains endroits connus d'eux seuls. Cette activité peut présenter des risques de dégradation du milieu et de ruptures d'équilibres biologiques, mais la réglementation reviendrait à poser la question du libre accès à la forêt domaniale.

35 La chasse reste l'activité qui entraîne le plus de conflits et pose le plus de problèmes. Les chausseurs se sont forgé un territoire mythique, qui leur appartient et qu'ils ont le droit de gérer en adhérant aux Sociétés de chasse. Dans le Parc, la réglementation autorise les propriétaires, les résidants permanents des communes incluses dans le Parc et un certain nombre d'autres personnes autorisées, à chasser à condition d'être inscrites à l'Association cynégétique du Parc. Les chasseurs forment un groupe de pression très important, n'hésitant pas à enfreindre la réglementation, en braconnant ou chassant en dehors des jours autorisés, menaçant même l'Administration lorsqu'elle ne cède pas à leurs exigences. Activité traditionnelle en Cévennes, nécessaire aux dires de chasseurs à l'équilibre des écosystèmes, la chasse est sans conteste le moins de friction le plus important entre les usagers et les pouvoirs chargés d'appliquer les règles de protection de l'environnement, car c'est en fait la traduction des luttes qui s'organisent pour la mainmise sur un territoire, réel pour les uns, car représentant une portion de l'espace productif, et mythique pour les autres, territoire de tous les pouvoirs, dont celui d'être un espace ludique. 
L'étude des différents acteurs intervenant dans la gestion ou ayant la jouissance d'un territoire est essentiellement faite sous l'angle des rapports de pouvoirs et des conséquences des interrelations des divers intervenants sur la dynamique de l'espace rural et en particulier l'évolution du milieu naturel. Poser cette problématique en termes de stratégies d'acteurs est relativement nouveau : cela ne pose pas de problème fondamental, mais reste cependant l'ambiguïté sémantique de ce concept face à des termes plus habituellement employés comme tactique ou politique. La territorialité des stratégies est beaucoup moins évidente à mettre en lumière, hors des stratégies foncières qui s'appliquent à des espaces parfaitement circonscrits. Il est cependant possible de considérer que chaque acteur de la dynamique des espaces possède son propre « territoire », même si celui-ci est mythique.

\section{RÉSUMÉS}

La démarche pour analyser les systèmes de pouvoirs qui interviennent dans la gestion des Parcs nationaux consiste tout d'abord en une identification des acteurs et de leurs pratiques. Il s'agit non seulement de les désigner, mais aussi de définir, pour la gestion des espaces, leur capacité réelle d'intervention, de déterminer le niveau de celle-ci et d'analyser les contradictions, voire les risques de conflits nés de leurs interrelations.

\section{Power structures and managment in the Cevennes National Park.}

The first task in any approach to the analysis of power structures in National Parks management is an identification of their relevant actors and their behaviour. One needs to define the capacity for such actors to enterine a spatial management acctivity and détermine both the level of their intervention and the extent to which the interaction of different actors promotes conflicts or mises contradictions.

\section{INDEX}

Keywords : Cévennes, power structures, National Park, State, Management

Mots-clés : systèmes de pouvoirs, Gestion, Parc national, État

\section{AUTEUR}

\section{MARIE-CLAUDE GUERRINI}

Chargée de recherche au CNRS, elle travaille sur l'évolution du milieu rural et plus particulièrement sur l'analyse de l'interface Homme/Milieu naturel. Elle a notamment collaboré au livre : Du rural à l'environnement. La question de la nature aujourd'hui.

N. MATHIEU, M. JOLLIVET (Dir.), (L'Harmattan, 1989). 